\title{
Journal of Artificial Organs 2019: the year in review
}

\author{
Journal of Artificial Organs Editorial Committee
}

\author{
Y. Sawa ${ }^{1} \cdot$ G. Matsumiya ${ }^{2} \cdot$ S. Miyagawa ${ }^{3} \cdot$ E. Tatsumi $^{4} \cdot$ T. Abe $^{5} \cdot$ K. Fukunaga $^{6} \cdot$ S. Ichiba $^{7} \cdot$ T. Taguchi $^{8} \cdot$ K. Kokubo $^{9}$. \\ T. Masuzawa ${ }^{10} \cdot$ A. Myoui ${ }^{11} \cdot$ M. Nishimura ${ }^{12} \cdot$ T. Nishimura ${ }^{13} \cdot$ T. Nishinaka ${ }^{14} \cdot$ E. Okamoto $^{15} \cdot$ S. Tokunaga ${ }^{16}$. \\ T. Tomo ${ }^{17} \cdot$ T. Tsukiya $^{4} \cdot$ Y. Yagi $^{18} \cdot$ T. Yamaoka $^{19}$
}

Received: 28 January 2020 / Published online: 19 February 2020

(c) The Japanese Society for Artificial Organs 2020

\section{Introduction}

Members of the Editorial Committee of the Journal of Artificial Organs (JAO) are pleased to introduce to colleagues worldwide through the publication of JAO a broad spectrum of important new achievements in the field of artificial organs. We believe that JAO has a very high potential for promoting interest in the field of artificial organs. The impact factor announced in the Journal Citation Reports for 2018 was 1.519 . We are proud of this impact factor, which will certainly enhance international interest in the journal.

Y. Sawa

sawa@surg1.med.osaka-u.ac.jp

1 Division of Cardiovascular Surgery, Department of Surgery, Osaka University Graduate School of Medicine, Osaka, Japan

2 Department of Cardiovascular Surgery, Chiba University Graduate School of Medicine, Chiba, Japan

3 Department of Cardiovascular Surgery, Osaka University Graduate School of Medicine, Osaka, Japan

4 Department of Artificial Organs, National Cerebral and Cardiovascular Center Research Institute, Osaka, Japan

5 Department of Urology, Iwate Medical University School of Medicine, Iwate, Japan

6 Faculty of Health Sciences, Kyorin University, Tokyo, Japan

7 Department of Surgical Intensive Care Medicine, Nippon Medical School Hospital, Tokyo, Japan

8 National Institute of Material Science, Ibaraki, Japan

9 Department of Medical Engineering and Technology, Kitasato University School of Allied Health Science, Kanagawa, Japan

10 Department of Mechanical Engineering, Ibaraki University, Ibaraki, Japan
From the beginning with Volume 1 in 1998 to the last issue (Volume 22) in 2019, we have received submissions from 43 countries, and we have accepted a total of 1140 papers for publication through the peer review process. In volume 22, we published 55 articles, amounting to 356 pages in total, including 36 original articles, 3 review articles, 11 case reports, and 5 brief communications. These papers were related to the many aspects of basic research, development, and clinical application of artificial organs, covering a variety of subfields. The yearly acceptance rate was $49.1 \%$ in 2019 . This review summarizes the content of some of the most intriguing papers of the last year.

11 Medical Center for Translational Research, Osaka University Hospital, Osaka, Japan

12 Division of Organ Regeneration Surgery, Faculty of Medicine, Tottori University, Tottori, Japan

13 Department of Therapeutic Strategy for Heart Failure, The University of Tokyo, Tokyo, Japan

14 Department of Cardiovascular Surgery, Tokyo Women's Medical University, Tokyo, Japan

15 Department of Human Science and Informatics, School of Bioscience and Engineering, Tokai University, Sapporo, Japan

16 The Department of Cardiovascular Surgery, JCHO Kyushu Hospital, Fukuoka, Japan

17 Second Department of Internal Medicine, Faculty of Medicine, Oita University, Oita, Japan

18 Department of Clinical Engineering, Kyoto Prefectural University of Medicine, Kyoto, Japan

19 Department of Biomedical Engineering, National Cerebral and Cardiovascular Center Research Institute, Osaka, Japan 
During the last year, a total of 134 reviewers who were specialists in artificial organs and interdisciplinary fields helped our authors to improve their manuscripts through thoughtful reviews, critiques, and suggestions. We are very happy to present such excellent work in JAO. We would like to express our profound gratitude to all authors, reviewers, and members from all over the world, and express the hope that you will continue to support our journal.

\section{Artificial heart (basic)}

For simultaneous optimization of the geometry of the impeller and volute in a centrifugal pump, Ghadimi et al. [1] proposed a fully automated optimization procedure using genetic algorithm. The algorithm consists of a geometry parameterization, CFD solver, artificial neural networks, and genetic algorithm module. They investigated three different scenarios using three different objective functions (1) hydraulic efficiency, (2) pressure head, and (3) hemolysis index (HI). From their results, at the same operating condition, the optimization scenario of hydraulic efficiency gives a considerable improvement in pump hydrodynamic efficiency with nearly the same level of $\mathrm{HI}$ reduction with respect to the optimization scenario for HI. Hence for reduction of computation time, the optimization of pump hydrodynamic efficiency is one of efficient way to design centrifugal pumps.

Interventricular dyssynchrony is caused by the shortening of the LV systole under continuous flow (CF) left ventricular assist device support (LVAD), and Shimamura et al. [2] investigated interventricular dyssynchrony on different CFLVAD support conditions. They measured stroke volume, stroke work, end-diastolic and end-systolic volumes, and end-systolic and end-diastolic pressure during LVAD support with different bypass rates $(0 \%, 50 \%, 75 \%$, and $100 \%)$. As the bypass rate increases from 0 to $100 \%$, the duration of interventricular dyssynchrony (DYS) continuously increases from 0 to $25.6 \pm 8.0 \%$. And increase of DYS causes decrease of stroke volume of the LV that is intimately connected with LV unloading. Their study is helpful for understanding interventricular interactions during the CF LVAD support, and this study will be expected to understand influences of the CF LVAD support on the right ventricular function.

One of the control methods of LVAD based on rotary blood pump (RBP) is a cardiac beat synchronization (CBS) modulating rotational speed in cardiac beat. Ogawa et al. [3] evaluated hemodynamics of the CBS system using a mathematical circulatory model including an RBP. The simulation demonstrated that systolic assist mode enhanced pulsatility in various cardiac conditions and diastolic assist mode reduced aortic insufficiency by extending AV opening interval when full bypass is avoided.

\section{Artificial heart (clinical)}

Matsumoto et al. [4] investigated the relationship between the externalizing direction of the driveline (the driveline angle) at the percutaneous exit site and driveline infection in patients with HeartMate II implantable LVAD. They retrospectively collected the data from 71 LVAD patients. The driveline angle was defined as the angle between a tangential line at the driveline exit site and a horizontal line, which measured on radiography in the supine position. The driveline infections were more frequently occurred in patients with driveline angle more than $42^{\circ}$. Multivariate analysis demonstrated that driveline angle more than $42^{\circ}$ is an independent risk factor for driveline infection. They concluded that the concept of driveline stability with adequate externalization at the exit site is very important for long-term durable driveline care.

Kawabori et al. [5] investigated the effect of performing a concomitant mitral valve procedure (MVP) during continuous-flow left ventricular assist device (CF-LVAD) implantation. Out of 526 patients who underwent primary LVAD implantation of a CF-LVAD, 108 patients had severe MR preoperatively. Of these 108 patients, 26 underwent a concomitant MVP and 82 did not. The results showed no difference between the two groups in all the parameters investigated, including overall survival, perioperative complications, and bridge-to-transplantation success.

Carrozzini et al. [6] reported several technical refinements to optimise the post-auricular (PA) driveline positioning for percutaneous power delivery of the Jarvik 2000 FlowMaker LVAD. In the study, the PA connection was managed through the "question mark-shaped" incision, the "C-shaped" incision, the "vertical incision" or the "orthogonal incision". They found that the implant technique resulted highly predictive of driveline infection, and concluded that the "orthogonal incision" was proposed and suggested to maximise the advantages of the Jarvik 2000 post-auricular driveline.

\section{Cardiopulmonary bypass}

Yasukawa et al. [7] The advantages of a non-invasive offpump CABG is that there is less damage to the heart, less blood destruction, and a higher possibility for a non-transfusion surgery. There may be many hospitals which would be interested to refer to this article focusing on aspirated blood. 


\section{Artificial lung/ECMO}

Assy et al. [8] have shown from their retrospective clinical studies that weaning from venovenous ECMO can be safely and smoothly achieved by combining NAVA (neurally adjusted ventilatory assist) mode in the treatment severe respiratory failure in children. They mentioned that the use of NAVA could allow the patient to adjust the ventilator setting by themselves, such as tidal volume, in response to the diaphragm's electorical activity.

Murphy et al. [9] through the retrospective analysis, reported that concurrent use of continuous renal replacement therapy (CRRT) in early stage during extracorporeal life support (ECLS) was associated with improved fluid balance and cytokine filtration. They concluded that early CRRT implantation may improve lung opacification observed on CXRs.

\section{Biomaterials}

Shukuzawa et al. [10] investigated the influences of the sealing length above the renal artery on gutter formation, nonapposed regions between the aortic wall, stent graft (SG), and chimney graft and incidence of flow channel to the aneurysm in chimney endovascular aortic aneurysm repair (Ch-EVAR) using a juxtarenal abdominal aortic aneurysm (AAA) model. Using micro-CT, the flow channel from the gutter to the aneurysm was successfully elucidated and influences of the SG size and the sealing length on the incidence of the flow channel were quantified. They suggests that the optimal sealing length should be chosen in consideration of the device design difference due to the device diameter in Ch-EVAR for juxtarenal AAA.

\section{Artificial valve}

Seki et al. [11] developed a novel polycaprolactone-made thermally deformable mitral annuloplasty ring and assessed its mechanical properties and the effect on the mitral valve anatomy. Results of the mechanical tests and animal tests, this new thermally deformable mitral annuloplasty ring effectively changed the coaptation length in all parts of the mitral valve and the distance from the coaptation to septum post-deformation via intraoperative heating.

Zhang et al. [12] examined the comparative efficacy and safety in $0.1 \mathrm{~mm}$ ePTFE and autologous pericardium in simplified hand-sewn trileaflet valved conduits implant surgery. They concluded that $0.1 \mathrm{~mm}$ ePTFE simplified hand-sewn trileaflet valved conduits show superiority to autologous pericardium valved conduits in terms of incidence of graft failure.

Kurumisawa et al. [13] examined whether sarcopenia may have close relationship with survival and hospital mortality after cardiovascular surgery in HD dependent patients. They concluded that sarcopenia evaluated by psoas muscle index have significant impact on long term survival rates in HD dependent patients undergoing cardiac surgery.

\section{Biomaterials}

Kumagai et al. [14] reported angiogenesis plays significantly important role in tissue regeneration in vivo. Some strategies have been reported to promote angiogenesis using vascular endothelial growth factors or other kinds of angiogenic factors. Herein, the authors evaluated angiogenesis and subsequent bone formation after implantation using a novel artificial bone named Affinos ${ }^{\circledR}$. Main component of this artificial bone is beta-tricalcium phosphate (beta-TCP) which is known as a biodegradable calcium phosphate. One of the advantage of using beta-TCP is supposed that calcium or phosphate ions after degradation of beta-TCP will become sources for new bone whose main component is hydroxyapatite (one of the calcium phosphates) and collagen. Also, other important characteristic is that this artificial bone has oriented porous structure. This structure enables to promote tissue infiltration including blood vessel when implanted in vivo. The authors implanted this artificial bone in the direction of the pores, parallel to the axis of the tibia and evaluated its angiogenic and bone formation properties. From histological observation, authors clarified that newly vessels and bone formed along the pores without using any growth factors. This finding suggests that angiogenic and bone formation could be controlled by changing micro structure of biomaterials.

\section{Tissue engineering/regenerative medicine}

Sakamoto et al. [15] evaluated recellularized acellular liver function with a blood extracorporeal circulation systema in rat hepatic failure model developed by combining $80 \%$ partial hepatectomy with warm ischemia. They showed the improved metabolism of ammonia in the blood and provided the good ex vivo system capable of evaluating the performance of recellularized grafts.

\section{Artificial kidney/dialysis}

Moerer et al. [16] examined the combination of continuous renal replacement therapy and minimal-flow extracorporeal $\mathrm{CO}_{2}$ removal to facilitate lung protective ventilation in patients with hypercarbia. $\mathrm{CO}_{2}$ removal and its effect on facilitation of lung-protective mechanical ventilation were assessed. The combined therapy reduced minute ventilation, tidal volume and $\mathrm{paCO}_{2}$, but no significant reduction in 
applied force was found, suggesting that the benefit of applying minimal-flow extracorporeal $\mathrm{CO}_{2}$ removal in an uncontrolled setting of mechanical ventilation might be limited.

Orcy et al. [17] carried out the cross-sectional study to validate among three direct dialysis quantification (DDQ) methods: total dialysate collection (TDC), continuous sampling of spent dialysate (CSSD), fractional sampling of dialysate (FSD) throughout 4hours hemodialysis sessions. The comparison between urea distribution volume (UDV) estimated by anthropometric method and through DDQ was a secondary outcome. The analysis was done through linear regression and Bland-Altman concordance method. They concluded that CSSD is simple, inexpensive, and needs small volume CSSD technique and this method can provide accurate information about the total amount of solutes removed by hemodialysis.

\section{Artificial liver, pancreas}

The optimal target blood glucose range after surgery is below $180 \mathrm{mg} / \mathrm{dL}$ and without hypoglycemia. However, the efficiency of tight control of serum glucose level within $110-180 \mathrm{mg} / \mathrm{dL}$ has not been unclear.

Conventionally, glycemic control mainly had used a sliding scale method. In recently, a closed-loop glycemic control device (STG-55) has been developed to maintain blood glucose levels within the target range through automatic infusion of insulin and glucose.

Tamura et al. [18] reported that using STG-55 to control blood sugar at $110-180 \mathrm{mg} / \mathrm{dL}$ reduced inflammation and complications after cardiovascular surgery.

Post-cardiovascular surgery patients divided into the STG-55 group who received closed-loop glucose control (target glucose level at 110-180 mg/dL) and the conventional group who received conventional glucose control using a sliding scale method (maintain blood glucose level $<200 \mathrm{mg} / \mathrm{dL}$ ).

They found that glucose control using closed-loop device might decrease inflammation after cardiovascular surgery without increasing hypoglycemia risk.

\section{Artificial skin, muscle, bone/joint, neuron}

Hip resurfacing arthroplasty (HRA) is attracting attention recently because HRA has several advantages such as preservation femoral bone stock, reduced risk of postoperative dislocation, good joint function, and easy revision if needed. Inoue et al. [19] investigated the mid-to long-term results of RHA in Japanese patients with osteoarthritis (OA) compared to those without and found better results in the patients with OA.
Sakai et al. [20] evaluated the difference in ADL after HRA, anterolateral total hip arthroplasty (ALTHA), and posterolateral THA (PL-THA) using self-completed questionnaire. Higher postoperative activities were suggested in patients with AL-THA and HRA patients compared with those with PL-THA.

\section{References}

1. Ghadimi B, Nejat A, Nourbakhsh SA, Naderi N. Shape optimization of a centrifugal blood pump by coupling CFD with metamodel-assisted genetic algorithm. J Artif Organs. 2018;1:29-36.

2. Shimamura J, Nishimura T, Mizuno T, Takewa Y, Tsukiya T, Inatomi A, Ando M, Umeki A, Naito N, Ono M, Tatsumi E. Quantification of interventricular dyssynchrony during continuous-flow left ventricular assist device support. J Artif Organs. 2018;4:269-75.

3. Ogawa D, Kobayashi S, Yamazaki K, Motomura T, Nishimura T, Shimamura J, Tsukiya T, Mizuno T, Takewa Y, Tatsumi E. Mathematical evaluation of cardiac beat synchronization control used for a rotary blood pump. J Artif Organs. 2018;4:276-85.

4. Matsumoto Y, Fukushima S, Shimahara Y, Yamashita K, Kawamoto N, Kuroda K, Seguchi O, Yanase M, Fukushima N, Shimizu $\mathrm{H}$, Kobayashi J, Fujita T. Driveline angle is crucial for preventing driveline infection in patients with HeartMate II device. J Artif Organs. 2018;1:37-433.

5. Kawabori M, Kurihara C, Conyer RT, Critsinelis AC, Sugiura T, Rosengart T, Morgan JA. Effect of concomitant mitral valve procedures for severe mitral regurgitation during left ventricular assist device implantation. J Artif Organs. 2018;2:91-7.

6. Carrozzini M, Bejko J, Gregori D, Gerosa G, Bottio T. How to implant the Jarvik 2000 post-auricular driveline: evolution to a novel technique. J Artif Organs. 2018;3:188-93.

7. Yasukawa T, Manabe S, Hiraoka D, Hirayama D, Kinoshita R, Komori M, Hosokawa M, Hirooka K. Safety and efficacy of a simple cardiotomy suction system as a blood salvage procedure during off-pump coronary artery bypass surgery. J Artif Organs. 2018;3:194-9.

8. Assy J, Mauriat P, Tafer N, Soulier S, EI Rassi I. Neurally adjusted ventilatory assist for children on veno-venous ECMO. J Artif Organs. 2018;2:118-25.

9. Murphy HJ, Eklund MJ, Hill J, Morella K, Cahill JB, Kiger JR, Twombley KE, Annibale DJ. Early continuous renal replacement therapy during infant extracorporeal life support is associated with decreased lung opacification. J Artif Organs. 2018;4:286-93.

10. Shukuzawa K, Akaoka T, Umezu M, Ohki T, Iwasaki K. Deployment of stent graft in an excessively higher position above the renal artery induces a flow channel to the aneurysm in chimney endovascular aortic aneurysm repair: an in vitro study. J Artif Organs. 2018;3:200-6.

11. Seki T, Jimuro K, Shingu Y, Wakasa S, Katoh H, Ooka T, Tachibana T, Kubota S, Ohashi T, Matsui Y. Mechanical properties of a new thermally deformable mitral valve annuloplasty ring and its effects on the mitral valve. J Artif Organs. 2018;2:126-33.

12. Zhang H, Ye M, Chen G, Jia B. $0.1 \mathrm{~mm}$ ePTFE versus autologous pericardium for hand-sewn trileaflet valved conduit: a comparative study. J Artif Organs. 2018;3:207-13.

13. Kurumisawa $\mathrm{S}$, Kawahito K. The psoas muscle index as a predictor of long-term survival after cardiac surgery for hemodialysisdependent patients. J Artif Organs. 2018;3:214-21.

14. Kumagai H, Makihara T, Funayama T, Sato K, Noguchi H, Abe T, Koda M, Yamazaki M. Angiogenesis and new bone formation in 
novel unidirectional porous beta-tricalcium phosphate: a histological study. J Artif Organs. 2018;4:294-9.

15. Sakamoto H, Shirakigawa N, Bual RP, Fukuda Y, Nakamura S, Miyata T, Yamao T, Yamashita YI, Baba H, Ijima H. A novel evaluation system for whole-organ-engineered liver graft by ex vivo application to a highly reproducible hepatic failure rat model. J Artif Organs. 2018;3:222-9.

16. Moerer O, Harnisch L-O, Barwing J, Heise D, Heuer JF, Quintel M. Minimal-flow ECCO2R in patients needing CRRT does not facilitate lung-protective ventilation. J Artif Organs. 2018;1:68-766.

17. Orcy RB, Antunes MF, Oses JP, Bohlke M. A new technique for low-volume continuous sampling of spent dialysate: a validation study. J Artif Organs. 2018;2:134-40.

18. Tamura T, Yatabe T, Namikawa T, Hanazaki K, Yokoyama M. Glucose control using a closed-loop device decreases inflammation after cardiovascular surgery without increasing hypoglycemia risk. J Artif Organs. 2018;2:154-9.
19. Inoue D, Kabata T, Kajino Y, Takagi T, Ohmori T, Yoshitani J, Ueno T, Tsuchiya H. Mid- to long-term results of resurfacing hip arthroplasty in Japanese patients: a comparison of osteoarthritic vs non-osteoarthritic patients. J Artif Organs. 2018;1:77-83.

20. Sakai T, Abe H, Nakamura N, Hamada H, Takao M, Sugano $\mathrm{N}$. Differences in activities of daily living after hip arthroplasty among hip resurfacing, anterolateral THA, and posterolateral THA: a propensity score matched analysis. J Artif Organs. 2018;1:84-90.

Publisher's Note Springer Nature remains neutral with regard to jurisdictional claims in published maps and institutional affiliations. 